\title{
BENCHMARKING COMO ESTRATÉGIA DE VANTAGEM COMPETITIVA
}

\author{
BENCHMARKING AS A COMPETITIVE ADVANTAGE STRATEGY
}

\begin{abstract}
Ana Paula Mendes Santos
Graduando em Administração pela Fundação Presidente Antônio Carlos de Teófilo Otoni-MG. E-mail: anapaulams.itaipe@gmail.com

Marcélia Aguiar Ferreira
\end{abstract}

Professora da Faculdade Presidente Antônio Carlos. Mestre em Administração Pública. Administradora. E-mail: marceliaaguiar@ hotmail.com Recebido: 16/06/2021 - Aceito: 17/06/2021

\section{Resumo}

Este estudo tem como propósito identificar a importância de utilizar o benchmarking, considerando as melhores práticas, resultando assim em um diferencial competitivo. Com tal propósito, esse trabalho foi desenvolvido a partir da metodologia de abordagem qualitativa e bibliográfica. Para tanto, o estudo aponta na sua pesquisa: história econceitos de marketing, conceitos de benchmarking, estratégias e vantagem competitiva do benchmarking. A pesquisa trata em adaptar a ferramenta nos processos das organizações, podendo identificar os pontos positivos de uma empresa se comparando com a outra, criando assim uma ferramenta de vital importância para o mercado competitivo.

Palavras-Chaves: Benchmarking; Estratégias; Vantagem Competitiva.

\begin{abstract}
This study aims to identify the importance of using benchmarking, considering best practices, thus resulting in a competitive differential. For this purpose, this work was developed based on the methodology of qualitative and bibliographic approach. To this end, the study points out in its research: history and marketing concepts, benchmarking concepts, strategies and competitive advantage os benchmarking. The research is about adapting the tool in the processes of organizations, being able to identify the positive points of one company comparing with the otler, thus creating a tool of vital importance for the competitive market.
\end{abstract}

Keywords: Benchmarking; Strategies; Competitive Advantage.

\section{Introdução}

Em um mundo dinâmico e competitivo, com constantes evoluções, as organizações buscam se diferenciar no mercado; por isso, elas tendem a caminhar junto 
Revista Multidisciplinar do Nordeste Mineiro, v.2

$2021 / 02$

ISSN 2178-6925

também se modificam, procurando sempre as melhores práticas, para que assim alcancem os resultados desejados e a superioridade competitiva. Hoje, para se mantiver 
firme no mercado é preciso estar atento a todas essas transformações, e é indispensável conhecer e saber empregar as ferramentas à disposição do administrador. Nesse sentido, o autor Spendolini $(1993$, p.3) já salienta que as organizações utilizando a técnica de benchmarking para se compararem com outras empresas, centrada nas premissas de que as estratégias promoveriam a melhoria continua em seus processos produtivos, focalizando o bom desempenho frente a concorrência.

Diante do exposto, é cada vez mais frequente o uso do benchmarking, uma ferramenta importante da administração estratégica, que tem trazido resultados favoráveis para as empresas. Neste contexto, o benchmarking além de ser indispensável, se mostra altamente relevante, por desafiar a empresa a buscar uma melhor versão de si todos os dias, não apenas copiando os processos dos concorrentes, mas ajustando à sua maneira prática de gestão em favor do potencial aumento de competitividade.

A partir dos relatos citados, fica evidente a importância do benchmarking para a gestão das organizações. Portanto, o presente trabalho tem como problemática: Qual a importância do benchmarking nas organizações como vantagem competitiva? Para responder esse questionamento, o artigo tem como objetivo mostrar o benchmarking como ferramenta na gestão a partir de um estudo bibliográfico. Proposto sob a busca minuciosa pela compreensão de mostrar a utilidade da ferramenta benchmarking num processo interno para que o sistema possa ser utilizado em boas práticas de um setor e tornar melhor os métodos administrativos da gestão.

Desse modo, o levantamento deve-se ao fato de a aplicação do benchmarking está se expandindo entre as grandes organizações e, sobretudo pelaespecificidade do tema como vantagem competitiva para as empresas que serão abordadas a seguir.

\section{Metodologia}

A metodologia adotada foi o levantamento de fontes bibliográficas em livros e artigos científicos. Pois, segundo Marconi e Lakatos (1992), a pesquisa bibliográfica é o levantamento de cada bibliografia já publicada, na forma de livros, revistas, publicações avulsas e empresa escrita. O seu propósito é fazer com que o 
pesquisador entre em familiaridade com todo o material escrito sobre determinado assunto. Ela pode ser encarada como o primeiro passo de toda a pesquisa científica.

Segundo o autor Gil (2010), a pesquisa bibliográfica é elaborada com material já publicado. Normalmente, esta modalidade de pesquisa inclui material impresso, como livros, revistas, jornais, teses, dissertações e anais de eventos científicos. Contudo, em virtude da disseminação de novos formatos de informação, estas pesquisas passam a conter outros tipos de fontes, como discos, fitas magnéticas, CDs, bem como o material disponibilizado pela internet.

\section{Referencial Teórico}

\subsection{História e Conceitos Do Marketing}

Historicamente é difícil identificar exatamente quando o marketing surgiu. Fato é que sua história está diretamente ligada à história da humanidade e sua evolução. "Marketing é uma disciplina nova de prática bem antiga" (MOTTA, 1983, p.37). Ou seja, desde os tempos em que as pessoas começaram a refletir sobre o comportamento das trocas, existe o pensamento sobre esse fenômeno. "É uma terminologia recente de uma prática, cujas origens remotas, aos primórdios da troca e comércio" (MOTTA, 1983, P.37). Quando não existiam moedas de fato, praticava-se o escambo, que era uma troca entre mercadorias de acordo com as necessidades das pessoas. Segundo Kotler (2000), desde as civilizações antigas havia, ainda que intuitivamente, pessoas com visão de marketing.

Segundo Cobra (1997, p.23), Marketing é “o processo na sociedade pelo qual a estrutura da demanda para bens econômicos e serviço é antecipado ou abrangidoe satisfeita através da concepção, promoção, troca e distribuição física de bens e serviços".

O marketing "é o processo social por meio do qual, pessoas e grupos de pessoas obtêm aquilo de que necessitam e o que desejam com a criação, oferta e livre negociação de produtos e serviços de valor com outros" (KOTLER, 2000, p.30). Já Richers (2000, p.5) sintetiza sua interpretação, definindo marketing como "a intenção de entender e atender o mercado". Podemos notar, pelos conceitos apresentados, que a 


\section{Revista Multidisciplinar do Nordeste Mineiro, v.2 $2021 / 02$ \\ ISSN 2178-6925}

visão de marketing atualmente busca o desenvolvimento de relações de longo prazo entre os envolvidos e seu conceito extrapola a comercialização de bens de consumo, incluindo ideias, estratégias, objetivos individual, programas organizacionais e sociais.

Para Chauvel (2001) o conceito de marketing surge no início do século XX, com foco na produção e na demanda, lidando com questões relacionadas à distribuição física dos produtos e aspectos econômicos e legais de transação, momento em que a economia de mercado foi se generalizando pelo mundo. $\mathrm{O}$ marketing foi um processo de desenvolvimento que acompanhou a própria evolução do mercado, criando novas perspectivas que o diferenciaram de outras práticas organizacionais, contando ainda, com as modificações feitas pelas escolas, que surgiram e se desenvolveram ao longo de sua existência. Com esse novo formato, houve transformações nas relações entre a economia e a sociedade, permitindo autonomia das atividades econômicas. As ligações que existiam entre produtores, trabalhadores e consumidores passaram, então, a ser regidas por leis de mercado e não mais pelo controle social, causando uma grande revolução no mercado, nas empresas e nos consumidores.

Para Las Casas (2001), Marketing é a área do conhecimento quecompreende todas as atividades concernentes ás relações de troca, orientadas para satisfação dos desejos e necessidades dos consumidores, visando atingir determinados objetivos de empresas ou indivíduos e considerando sempre o meio ambiente de execução e impacto que essas relações causam no bem-estar da sociedade.

Kotler (2003) prescreve que marketing é a função empresarial que identifica necessidades e desejos insatisfeitos, define e mede sua magnitude e seu potencial de rentabilidade, especifica que mercados-alvo serão mais bem atendidos pela empresa, decide sobre produtos, serviços e programas adequados para servir a esses mercados selecionados e convocados a todos na organização para pensar no cliente e atender ao cliente. Kotler (2003) ainda ressalta que, o marketing ostenta uma rica variedade de conceitos e ferramentas, sendo uma resposta relacionada à maneira de competir em outras bases que não apenas o preço, tornando-se mais importante do que nunca,convertendo as necessidades cambiantes das pessoas em oportunidades lucrativas paraas empresas.

Limeira (aput DIAS et al., 2006, p.2), por sua vez, apresenta o conceito de Marketing como: 
[...] a função empresarial que cria continuamente valor paara o cliente e gera vantagem competitiva duradoura para a empresa, por meio da gestão estratégia das variáveis controláveis de Marketing: produto, preço, comunicação e distribuição.

Segundo Kotler e Koller (2006, p.4) "Marketing envolve a identificação e a satisfação das necessidades humanas e sociais" sendo definido de uma maneira simplificada pelo autor "como forma de suprir necessidades lucrativamente".

Contudo, Ambler afirma que:

O marketing tem existido desde o início do comércio, sempre pensando, mas não era chamado desta forma. Mercadores não simplesmente compravam e vendiam; eles desenvolviam relacionamentos de longo prazo, o que agora seria chamado de equidade da marca. Eles podem não ter sido introspectivos sobre seus métodos de negócios, mas se eles tivessem conhecimento de como satisfazer seus consumidores, enquanto faziam lucro para eles mesmos, o comércio não teria sobrevivido (AMBLER, 2014 aputSANTOS, 2009).

Em contraponto a Santos (2009), Chauvel (2001) já data o início do marketing no começo do século XX, contextualizando que a economia de mercado era predominante no mundo ocidental. Esse sistema mudou as relações entre a economia e asociedade, dando autonomia às atividades econômicas. Assim, as leis de mercado, e nãoo controle social iriam reger as relações entre produtores, trabalhadores e consumidores. Por fim, complementa que, da ruptura entre a organização social e a produção, surgiu o aparecimento de uma necessidade até então nula: a de se estabelecer uma relação entre produtores e compradores.

O marketing envolve a identificação e a satisfação das necessidades humanas w sociais. Para defini-lo de uma forma mais simples, podemos dizer que ela supre as necessidades de forma lucrativa (KOTLER, 2013).

\section{Conceitos De Benchmarking}

Spendolini (1993) descreve benchmarking como um processo de investigação continua; um processo de aprendizado com ouros, que busca ideias 


\section{Revista Multidisciplinar do Nordeste Mineiro, v.2 $2021 / 02$ \\ ISSN 2178-6925}

pragmáticas e que leva tempo, mas, é viável e fornece informações úteis para a melhoria de qualquer atividade empresarial. Dessa forma, Spendoline (1993) deixa claro que benchmarking não é copiar, não é imitar, e, não é uma ferramenta que se aplica uma única vez e fornece respostas simples.

Camp (1998) afirma que o Benchmarking é o processo duradouro que busca novas ideias, práticas e processos que tem como base boas características, que a sua implementação tem como objetivo obter o melhor resultado em cima dos dados obtidos. Essa pesquisa industrial ou levantamento de dados leva o gerente a comparar o desempenho de sua empresa com o desempenho de outras empresas.

Num tempo em que a dinâmica das relações empresariais vão passando por novos processos de atualização e tomada de decisões, no entendimento de Maximiano (2004), benchmarking refere-se a técnica de comparação do desempenho de uma empresa com a de outra.

Araújo (2006, p.235) faz a seguinte afirmação sobre o benchmarking:

É uma abordagem de gestão organizacional que conduz a tão desejada excelência, utilizando-se de procedimentos de investigação que pretendem reunir e adaptar as respostas encontradas por outras organizações. Longe de se igualar à meia cópia, revela alternativas valiosas de incrementos dos níveis de eficácia e eficiência.

Em uma explicação rápida, Chiavenato (2007, p.340), define o benchmarking como "uma forma de estudar e analisar o que outras empresas estão fazendo de melhor".

Para o autor Chiavenato (2008, p.196), “o benchmarking é um processo contínuo para avaliar produtos, serviços e práticas dos concorrentes mais fortes e das empresas que são reconhecidas como líderes empresariais, com o propósito de aprimoramento empresarial". O processo de benchmarking verifica o que os melhores concorrentes estão fazendo e observa suas práticas comerciais, a fim de partir para o que de melhor existe.

Benchmarking é uma técnica ou ferramenta de gestão que envolve tomar como referência os melhores aspectos ou prática de outras empresas, seja de competidor 


\section{Revista Multidisciplinar do Nordeste Mineiro, v.2 $2021 / 02$ \\ ISSN 2178-6925}

direto ou de pertencentes outro setor (e, em alguns casos, de outras áreas da empresa), e adapta-se à empresa, acrescentando melhorias (CAMP, 2008).

De acordo com Araújo (2009, p.196) "benchmarking é uma das tecnologias de maior utilidade para a gestão organizacional, centrada na premissa de que é imperativo Explorar, compreender, analisar e utilizar as soluções de uma organização, concorrente ou não, frente a determinado problema”.

Benchmarking é o processo contínuo de medir produtos, serviços e práticas de concorrentes reconhecidos como líderes em seu setor (SILVA; FONSECA, 2009).

O benchmarking é um processo de comparação de produtos, práticas e serviços de uma empresa considerada como forte concorrente ou reconhecida por ser melhor no mercado. Ela se dá através de uma pesquisa que compara os processos e práticas de cada empresa, identificando os pontos fortes de cada uma, destacando o que pode ser utilizado para sua empresa a fim de se alcançar uma vantagem a nível superior na competitividade do mercado (BERNARDES, 2014).

\section{Estratégias Do Benchmarking}

Para Spendolini (1994) há um ponto a ser destacado é que as organizações que começam o processo de benchmarking com o propósito ou objetivo claro têm maior sucesso do que aquelas que empreendem em esforço de benchmarking sem um senso de propósito ou uma direção. O autor Spendolini (1994) ainda afirma que algumas organizações usam o benchmarking como uma variedade de propósitos e o posicionam como parte de um processo gral de solução de problemas, com obrigação clara de aperfeiçoamento organizacional.

Zairi e Leonard (1995) afirmam que:

O benchmarking é usado em nível estratégico para se determinarem padrões de desempenho com relação a quatro prioridades corporativas: satisfação do consumidor; motivação e satisfação do funcionário, participação de mercado; retorno sobre ativos.

Segundo Gomes (2004), entende-se benchmarking como uma procura por melhores atividades estratégicas que conduzem uma organização a fomentar seus 
negócios empresariais, baseando-se em práticas já utilizadas, reformuladas para obter melhores resultados entre os demais.

O benchmarking, como se pode notar, é uma estratégia em que se procura aprender com outras empresas, fator que requer tempo e disciplina. Essa ferramenta pode ser aplicada em qualquer processo e é importante para qualquer empresa, já que se trata de um instrumento que contribuiu para o melhoramento do desempenho da empresa (VASCONCELOS; CANEN; LINS, 2006).

\section{Vantagem Competitiva}

Benchmarking é um processo positivo, pró-ativo e estruturado que conduz a mudanças, ao desempenho e á vantagem competitiva. Esse processo busca novas ideias para métodos, práticas e processos param então adotá-las e praticá-las. É a busca das melhores práticas na indústria que conduzirão ao desempenho superior e ao alcance da eficácia global (CAMP, 1993).

Segundo os autores Zairi e Leonard (1995, p. 246-248), a única forma de manter a vantagem competitiva e de atingir superiores níveis de desempenho é através das comparações externas. [...] O benchmarking refere-se basicamente ao atingimentode desempenho superior em longo prazo. Em curto prazo, todavia, ele pode referir-se ao frenético tentar eliminar as lacunas negativas entre as organizações e seus concorrentes. Este, porém, não é o fim do benchmarking, o agir frenético não vai levar a superioridade. As organizações devem compreender que, uma vez que as lacunas sejam eliminadas, elas devem estar na posição de suplantar a concorrência e manter a superioridade por longo prazo.

E reforçam que: "benchmarking refere-se à proteção da vantagem competitiva, mas também à recuperação dos processos fracos, através de seu fortalecimento por meio de novos aprendizados, novas práticas, novos métodos e novas técnicas" (ZAIRI; LEONARD, 1995, p. 246-248).

De acordo com Boxwell (1996, p. 21) o benchmarking proporciona à organização uma vantagem competitiva e um desempenho superior com relação à concorrência. 


\section{Revista Multidisciplinar do Nordeste Mineiro, v.2 $2021 / 02$ \\ ISSN 2178-6925}

Camp (1998) afirma que, entre diversas outras formas de abordagem, o Benchmarking é o processo, estruturado e pró-ativo conduzindo a mudanças nas operações e no desempenho superior e levando a uma vantagem competitiva, assim como a investigação e incorporação de melhores práticas da indústria leva a um maior giro de estoque e retornos sobre ativos altos e lucrativos.

Segundo Martin (2002), a Xerox foi uma das primeiras empresas a adotar o benchmarking, ao utilizá-lo como ferramenta para atingir uma vantagem competitiva. Já, Spendolini (1992), afirma que o benchmarking é um sistema de pesquisa que consiste na avaliação dos processos de trabalho nas empresas que são reconhecidas como representantes das melhores práticas, com o objetivo de se atingir o aprimoramento organizacional. Dessa forma, propicia uma comparação de processos e práticas administrativas entre as organizações que buscam alcançar a vantagem competitiva e a busca pela Gestão da Qualidade Total.

“É o processo de pesquisa que possibilita aos gestores desenvolver comparações entre empresas para identificar o 'melhor dos melhores', e, assim, obter um nível de superioridade e vantagem competitiva" (CAMP, 2008, p. 15).

Para Araújo (2009), o benchmarking proporciona a uma organização informações que lhe sirvam de referencial em sua trajetória de busca eterna pela qualidade. As grandes organizações investem em benchmarking e treinam seu corpo funcional para conduzir uma investigação e o estimulam a participar do processo.

Maximiano (2011) enfatiza que o importante é reconhecer que benchmarking é um processo não só para obter metas métricas quantificáveis, mas também para investigar e documentar as melhores práticas da indústria, as quais permitem que se atinjam as metas.

\section{Considerações Finais}

Em síntese, o benchmarking é reconhecido como ferramenta para comparar produtos, serviços e empresas, rente aos seus processos em relação às outras empresas. Para tanto, esta técnica além de assessorar os gestores, propõe detectar e atuar com processos de melhoria continua nas organizações, alicerçando as empresas que almejam 
o alcance da qualidade segundo suas necessidades. De forma intermitente, eles devem observar os pontos fortes e fracos que as empresas concorrentes desempenham,usandoas como referência, para se diferenciar no mercado.

Este estudo propôs-se a responder o problema de pesquisa citado na introdução: Qual a importância de se utilizar o benchmarking nas organizações como vantagem competitiva? Em que os objetivos foram: mostrar o benchmarking como ferramenta na gestão a partir de estudo bibliográfico. Proposto sob a busca minuciosa pela compreensão de mostrar a utilidade da ferramenta benchmarking num processo interno para que o sistema possa ser utilizado em boas práticas de um setor e tornar melhor os métodos administrativos da gestão.

Segundo Chiavenato (2008), o benchmarking é uma fonte inesgotável de ideias e experiências alheias. Este conceito significa um marco de referência, um padrão de excelência que precisa ser identificado para servir de base para mudanças inovação, pois permite comparações de processos e práticas entre empresas para identificar o que há de melhor e alcançar um nível de superioridade ou vantagem competitiva.

Concluímos então, que o benchmarking dentro de uma organização é fonte de vantagem competitiva, pois alastra o conhecimento na empresa, estando relacionado diretamente ao processo de melhoria continua. Através dessa ferramenta, as organizações podem descobrir novas oportunidades de melhoria continua e estabelecer uma cultura de aprendizagem continua de novas práticas.

Compreendemos a importância do uso do processo do benchmarking nas organizações, seja elas de pequeno, médio ou grande porte. Porém é preciso esta atenta em tais processos, pois nem sempre as empresas vão se adaptar as mesmas práticas.

\section{Referências}

ARAÚJO, Luiz César G. Gestão de Pessoas - Estratégias e Integração Organizacional. São Paulo: Atlas, 2006. de. Organizações, sistemas e métodos e as tecnologias de gestão organizacional: arquitetura organizacional, benchmarking, empowerment, gestão pela qualidade total, reengenharia: volume 1. 4. Ed. 2. Reimpressão. São Paulo: Atlas, 2009. 


\section{Revista Multidisciplinar do Nordeste Mineiro, v.2 2021/02 \\ ISSN 2178-6925}

BERNARDES, Vania Marisa da Mota. O benchmarking como ferramenta de gestão para a indústria de calçados. 2014. Disponível em:

http://repositorio.uportu.pt/xmlui/bitstream/handle/11328/1218/TMG\%2014.pdf?sequen $\underline{\text { ce }=4 \& \text { is Allowed }=y}$. Acessado em: 15 de Abril de 2018.

BOXWELL, R. J. Vantagem competitiva através do benchmarking. São Paulo: Makron Books, 1996.

CAMP, R. C. Benchmarking: o caminho da qualidade total. São Paulo: Pioneira, 1993.

Benchmarking: o caminho da qualidade total. São Paulo: Pioneira, 1998.

. Benchmarking: o caminho da qualidade total. São Paulo: Pioneira, 2008.

CHAUVEL, L. "Uma nova era da sociedade americana? Dinâmica e Perspectivas da Estrutura Social nos Estados Unidos (1950-2000)", OFCE Review, N. 76, PP.751. 2001.

CHIAVENATO, Idalberto. Administração: teoria, processo e prática. 4. Ed. Rio de Janeiro: Elsevier, 2007.

Empreendedorismo: dando asas ao espírito empreendedor: empreendedorismo e viabilização de novas empresas: uma guia eficiente para iniciar e tocar seu próprio negócio. 3. Ed. São Paulo: Saraiva, 2008.

COBRA, Marcos. Marketing básico: uma perspectiva brasileira. 4. Ed. São Paulo: Atlas, 1997.

DIAS; Sérgio Roberto et al. Marketing: estratégia e valor. São Paulo: Saraiva, 2006. GIL, Antônio Carlos. Como Elaborar Projetos de Pesquisa. $5^{\text {a }}$ ed. São Paulo: Atlas, 2010 .

GOMES, Carlos Francisco Simões. Gestão da Cadeia de Suprimentos integrada à Tecnologia da Informação. São Paulo: Pioneira Thomson Learning, 2004. Disponível em: 
Revista Multidisciplinar do Nordeste Mineiro, v.2

$2021 / 02$

ISSN 2178-6925

HTTP://books.google.com.br/books?id=B06QoZ8jB8IC\&dq=benchmarking+conceito \&hl=pt-

$\underline{\text { BR\&sa }=X \& e i=6 b z N U a O}$ OrOC0QHPI4HIDg\&ved=0CDgQ6AEwAQ\#v=onepage \&q $=$ benchmarking $\% 20$ conceito $\& \mathrm{f}=$ false. Acessado em: 25.06.2013.

KOTLER, P. Administração de Marketing. 10. Ed. São Paulo: Prentice hall, 2000. Marketing de A a Z: 80 conceitos que todo profissional precisa saber. 3. Ed. Rio de Janeiro: Campus, 2003.

KOTLER, Philip e Keller, Kevin Lane. Administração de marketing. Tradução Mônica Rosenberg, Brasil Ramos Fernandes, Cláudia Freire, revisão técnica Dilson Gabriel dos Santos. $12^{\circ}$ edição. São Paulo: Pearson Prentice Hall, 2006.

. Marketing essencial: conceitos, estratégias e casos. 5. Ed. São Paulo: Pearson Education do Brasil, 2013.

LAS CASAS, A. L. Marketing: uma introdução. In: Marketing: Conceitos, Exercícios e Casos. São Paulo: Editora Atlas, 2001.

MARCONI, Marina de Andrade; LAKATOS, Eva Maria. Metodologia do trabalho científico. São Paulo: Editora Atlas, 1992. 4ª ed. p.43 e 44.

MAXIMIANO, A. C. A. Introdução à administração. 6. Ed. São Paulo: Atlas, 2004. Teoria Geral da Administração: da revolução urbana à revolução digital - 6. Ed. reimpr. - São Paulo: Atlas, 2011.

MOTTA, F. C. P. A questão da formação do administrador. ERA - Revista de Administração de Empresas. FGV, Rio de Janeiro, v. 23, n. 4, Out./Dez. 1993. RICHERS, Raimar. Surfando as ondas do mercado. São Paulo: Negócio Ed., 2000. SANTOS, T.; LIMA, M. V. V.; BRUNETTA, D. F.; FABRIS, C.; SELEME, A. O desenvolvimento do Marketing: uma perspectiva histórica. Revista de Gestão, v. 16, n. 1, p. 89-102, 2009. Disponível em: http://www.regeusp.com.br/arquivos/767.pdf. Acessado em 14 abr. 2014. 
Revista Multidisciplinar do Nordeste Mineiro, v.2 $2021 / 02$

ISSN 2178-6925

SILVA, A. S.; FONSECA, A. C. P. D. Controle gerencial por meio do benchmarking: o caso de uma organização de ciência e tecnologia da Marinha do Brasil. Contabilidade Vista \& Revista, v. 20, n. 2, p. 65-98, 2009.

SPENDOLINI, Michael J. Benchmarking. 1. ed. São Paulo: Mkron Books, 1992.

Benchmarking. Tradução Katia Aparecida Roque. Revisão técnica Eduardo Correa Moura. - São Paulo: Ed. Makron Book. 1993.

. Benchmarking. São Paulo: Amacom, 1994.

VASCONCELLOS, Vinícius Albuquerque; CANEN, Alberto Gabbay; LINS, Marcos Pereira Estellita. Identificando as melhores práticas operacionais através da associação Benchmarking - Dea: o caso das refinarias do petróleo. Pesqui. Oper., v. 26, n. 1, p.5167, 2006.

ZAIRI, M.; LEONARD, P. Benchmarking prático: o guia completo. Tradução de Maria Teresa Corrêa Oliveira. São Paulo: Atlas, 1995. 